\title{
Psicooncología
}

ISSN: 1696-7240

\section{La comunicación entre el paciente oncológico y los profesionales. El Cuestionario de Comunicación de la EORTC ${ }^{1}$}

\author{
Juan Ignacio Arraras ${ }^{2 *}$; Lisa M. Wintner ${ }^{3}$; Monika Sztankay ${ }^{3,4}$; Krzysztof Tomaszewski ${ }^{5}$; Dirk \\ Hofmeister $^{6}$; Anna Costantini ${ }^{7}$; Anne Bredart ${ }^{8}$; Teresa Young ${ }^{9}$; Karin Kuljanic ${ }^{10}$; Iwona M. \\ Tomaszewska ${ }^{11}$; Meropi Kontogianni ${ }^{12}$; Wei-Chu Chie ${ }^{13}$; Dagmara Kullis ${ }^{14}$; Eva Greimel ${ }^{14}$; \\ Uxue Zarandona ${ }^{15}$
}

Recibido: 15 de marzo de 2017 / Aceptado: 15 de abril de 2017

Resumen. Este trabajo pretende introducir el área de la comunicación entre el paciente oncológico y los profesionales, y destacar el impacto que tiene en el paciente. Además, se presenta el cuestionario de comunicación de la EORTC. La comunicación entre el paciente y los profesionales es uno de los elementos claves del soporte que se ofrece a dichos pacientes. En dicha comunicación participan un rango

Realizado en nombre del Grupo de Calidad de Vida de la EORTC.

Servicios de Oncología. Complejo Hospitalario de Navarra. Pamplona.

3 Department of Psychiatry and Psychotherapy; Innsbruck Medical University; Austria.

E-mail: monika.sztankay@tirol-kliniken.at

4 Leopold-Franzens-University Innsbruck; Austria.

E-mail: lisa.wintner@student.uibk.ac.at

5 Health Outcomes Research Unit; Department of Gerontology, Geriatrics, and Social Work; Faculty of Education; Ignatianum Academy; Krakow; Poland.

E-mail:krtomaszewski@gmail.com

6 University of Leipzig; Leipzig; Germany.

E-mail: Dirk.Hofmeister@medizin.uni-leipzig.de

7 Psycho-Oncology Unit; Sant'Andrea Hospital; Faculty of Medicine and Psychology; Sapienza University; Rome. Italy.

E-mail: annacostantini@alice.it

8 Institut Curie. Paris. France.

E-mail: anne.bredart@curie.net

9 Mount Vernon Cancer Centre; Northwood; UK.

E-mail: teresa.young2@nhs.net

10 Department of Gynecology and Obstetrics. University Hospital Center Rijeka. Croatia.

E-mail: karin.kuljanic@kbc-rijeka.hr

11 Department of Medical Education; Jagiellonian University Medical College; Krakow; Poland.

E-mail: im.tomaszewska@gmail.com

12 Department of Nutrition \& Dietetics; Harokopio University. Athens; Greece.

E-mail: mkont@hua.gr

13 Department of Public Health; Institute of Epidemiology and Preventive Medicine; College of Public Health; National Taiwan University; Taiwan.

E-mail: weichu@ntu.edu.tw

14 EORTC QL Department. Brussels.

E-mail: dagmara.kulis@eortc.be

15 Medical University Graz. Austria.

E-mail: elfriede.greimel@klinikum-graz.at

* Dirección de correspondencia: Juan Ignacio Arraras. Servicios de Oncología. Complejo Hospitalario de Navarra. Irunlarrea 3; 31008. Pamplona. España. E-mail: jiarraras@correo.cop.es 
importante de profesionales. Hay una necesidad de realizar más investigación sobre la comunicación. Se presentan dos modelos principales de atención al paciente: el Paternalista y el de Atención Centrada en el Paciente con cáncer. Este último lleva asociada la Comunicación Centrada en el Paciente - CCP. Se revisa la relación entre comunicación y otros PRO: Calidad de Vida, información, y Satisfacción con los Cuidados. Existen diferencias culturales en comunicación que pueden estar relacionadas con el modelo de atención al paciente. El Grupo de Calidad de Vida de la Organización Europea para la Investigación y Tratamiento del Cáncer-EORTC está desarrollando una escala de comunicación entre el paciente oncológico y los profesionales. La mayoría del contenido de dicho cuestionario se centra en las conductas de los profesionales. Los aspectos culturales tienen un papel fundamental en el desarrollo del instrumento. El cuestionario se basa en el modelo de Comunicación Centrada en el Paciente CCP. Se presenta el cuestionario EORTC QLQ-COMU26, que consta de seis escalas y cuatro ítems individuales. Se describen las tres primeras fases que se han dado en su creación. En la actualidad su funcionamiento psicométrico se está valorando en un estudio internacional.

Palabras clave: Comunicación; EORTC; cuestionario; oncología.

\title{
[en] The communication between cancer patients and professionals. The EORTC communication questionnaire
}

\begin{abstract}
The aims of the present work are to introduce to the field of communication between the cancer patient and the professionals, to remark the positive influence communication may have on the patient, and to present the EORTC communication questionnaire. Communication between patient and professional is a key element in the support that is offered to cancer patients. It is important to consider different professionals communicate with cancer patients. There is a need of research in communication between patients and professionals. Two main models of patient care are presented: Paternalistic and Patient-Centered Cancer Care. Patient-Centered Care includes Patient- Centered Communication - PCC. The relation between communication and other PROs - Quality of Life, Information and Satisfaction with Care - is presented. There are cross-cultural differences in communication that could be related to the model of patient care. The European Organisation for Research and Treatment of Cancer (EORTC) Quality of Life Group is developing a questionnaire to assess communication between cancer patient and the professionals. This Communication questionnaire mainly assesses professionals' behaviors. Cultural aspects have a key role in the development of the EORTC questionnaire. This instrument is based on the Patient-Centered Communication - PCC model. The EORTC QLQ-COMU26 is presented. It includes six scales and four individual items. The three phases of the questionnaire development process are described. At the present moment the EORTC QLQ-COMU26 is being field-tested in a larger international study (phase IV), to ensure it is an appropriate and psychometrically valid instrument.
\end{abstract}

Keywords: Oncology; communication; questionnaire; EORTC.

Sumario. 1. Introducción: la comunicación en oncología entre pacientes y profesionales 2. Necesidad de investigar en comunicación entre pacientes y profesionales 3. Modelos de cuidados 3.2. Modelo Paternalista 3.2.1. Comunicación dentro del modelo Paternalista 3.3. Atención Centrada en el Paciente con Cáncer y Comunicación Centrada en el Paciente 3.4. Ventajas de la CCP 3.5. Comunicacion y otros pro (Patient Reported Outcomes) 3.6. Comunicación y Calidad de Vida 3.6.1. Comunicación e información 3.7. Comunicación y satisfacción con los cuidados 3.8. Diferencias culturales en comunicación 4. El cuestionario de comunicación de la EORTC 4.1. Objetivos del cuestionario 4.2. Utilidad 4.3. Desarrollo del cuestionario de comunicación 4.4. Cuestionario de comunicación de la EORTC QLQ-COMU26: contenido y población a la que se dirige 4.5. Obtención del cuestionario actual de comunicación 5. Conclusiones 6. Agradecimientos 7. Referencias bibliográficas.

Cómo citar: Arraras JI, Wintner LM, Sztankay M, Tomaszewski K, Hofmeister D, Costantini, et al. La comunicación entre el paciente oncológico y los profesionales. El cuestionario de comunicación de la EORTC. Psicooncologia 2017; 14: 107-20. DOI: 10.5209/PSIC.55815 


\section{Introducción: la comunicación en oncología entre pacientes y profesionales}

La comunicación entre el paciente y los profesionales es un elemento fundamental en el campo de la Oncología. Es considerada una habilidad clínica clave ${ }^{(1-3)}$. La comunicación efectiva en Oncología se ha definido como un diálogo entre el paciente y el equipo, donde la información se transmite de manera satisfactoria, y ninguna de las partes se ve restringida al dar sus opiniones, preguntas o preocupaciones ${ }^{(4)}$.

Dentro del proceso de comunicación en oncología, hay un número importante de personas que pueden tomar parte: pacientes, cuidadores (familiares y otros), profesionales, y además otras fuentes de información, como los medios. En el presente artículo y en el cuestionario de la EORTC de comunicación nos vamos a centrar en la comunicación entre los pacientes y los profesionales.

La comunicación entre el paciente y los profesionales se reconoce como uno de los elementos principales del soporte que se ofrece a los pacientes ${ }^{(5)}$, en especial a los de enfermedad avanzada ${ }^{(6)}$. Un buen sistema de comunicación entre paciente y profesionales puede mejorar la vivencia que tiene el paciente de los tratamientos oncológicos $^{(7)}$. Puede además ayudar a mejorar síntomas ${ }^{(8)}$, como el dolor o el funcionamiento emocional, y la Calidad de Vida en general ${ }^{(9)}$.

La comunicación tiene un papel fundamental al dar malas noticias. La comunicación entre el paciente y el profesional se puede entender como un proceso en el que sus componentes se influyen unos a los otros. En este sentido, dar y recibir malas noticias requiere que se haga dentro de un proceso de comunicación de doble dirección ${ }^{(10)}$.

La comunicación entre los pacientes y los profesionales puede explorar y ayudar al paciente a determinar sus preferencias sobre los cuidados de salud y ayudar a los profesionales a entender estas preferencias ${ }^{(11)}$.

Al evaluar la comunicación entre el paciente y los profesionales se aconseja tener en cuenta que las necesidades de los pacientes en relación a la comunicación, y al soporte en general, pueden cambiar a lo largo del proceso de tratamiento ${ }^{(12,13)}$.

En la atención al paciente oncológico participan un rango importante de profesionales. Todos ellos tienen un papel fundamental en el soporte que se ofrece a los pacientes, ya que cada uno de ellos puede mantener (en menor o mayor medida) comunicación con el paciente, además de manejar información relacionada con su papel profesional. Es adecuado que los diferentes profesionales se comuniquen de manera adecuada con el paciente ${ }^{(14)}$.

\section{Necesidad de investigar en comunicación entre pacientes y profesionales}

La comunicación entre los pacientes y los profesionales es un área importante de investigación. En la actualidad se recomienda realizar más investigación sobre este campo, ya que una gran parte de las necesidades no resueltas referidas por los pacientes se centran en la comunicación con los profesionales ${ }^{(15)}$. Carlson et al. $(2005)^{(16)}$ entienden que hace falta más investigación que defina los elementos que contribuyen a una comunicación satisfactoria dentro de la atención de Salud. Comprender cómo optimizar el proceso de comunicación entre los pacientes y el equipo que atiende, puede ser esencial para reducir la carga producida por el cáncer ${ }^{(17)}$.

Un área a la que se le presta cada vez más esfuerzos es la del entrenamiento a los profesionales de salud en comunicación con el paciente oncológico, y en la evaluación 
de la efectividad de dicho entrenamiento ${ }^{(4)}$. Puede ser de utilidad ver la percepción que tienen los propios pacientes de la efectividad de dicha formación. Es deseable que dicho entrenamiento se inicie dentro de los propios estudios universitarios, y se mantenga a lo largo de la formación post-grado y la vida profesional.

\section{Modelos de cuidados}

Los modelos de cuidados indican el tipo de relación que se establece entre los pacientes y los profesionales. Estos modelos incluyen conductas, expectativas y actitudes, e influyen en el tipo de comunicación que los pacientes y los profesionales pueden establecer. En la literatura se recogen dos modelos principales de atención: Paternalista y el de Atención Centrada en el Paciente con cáncer, y a ellos nos vamos a referir. Es cierto que se han tenido en cuenta algunos otros modelos de cuidados en la literatura: Morgan ${ }^{(18)}$ por ejemplo, describe una relación de consumidor en la que el paciente toma un papel activo y el médico un papel tendente a la pasividad (aceptando las peticiones de los pacientes); y una relación de vacío, en la que los pacientes siguen adoptando un rol pasivo incluso cuando los médicos reducen parte de su control. Podríamos decir que a las consultas de este modelo de vacío les puede faltar suficiente dirección.

\subsection{Modelo Paternalista}

Parsons ${ }^{(19)}$ fue uno de los primeros sociólogos en estudiar la relación entre los pacientes y los profesionales. Este autor describió el modelo paternalista como el de una relación asimétrica en la que el profesional ocupaba una posición dominante debido a su nivel de conocimientos. Este sistema se veía como la única forma de relación entre médico y paciente. Dentro de este modelo, Parsons describió el rol del enfermo, en el cual el paciente se veía como alguien que necesita cuidados médicos y que básicamente debe cooperar con el profesional. El profesional tenía el rol complementario: ofrecía sus conocimientos y habilidades para ayudar al paciente, y tenía bastante autonomía a la hora de ejecutar sus habilidades profesionales.

\subsubsection{Comunicación dentro del modelo Paternalista}

Los profesionales dentro del modelo paternalista tienden a centrar su atención en los aspectos físicos de la enfermedad del paciente. Las preguntas que plantean son principalmente de un estilo cerrado, como: “'ha tenido dolor?” Estas preguntas buscan obtener información que ayude al profesional a entender la enfermedad dentro de su modelo biomédico, y dan pocas oportunidades al paciente para expresar sus propias creencias y preocupaciones, o hablar de otros aspectos no propuestos por el profesional ${ }^{(18)}$.

\subsection{Atención Centrada en el Paciente con Cáncer y Comunicación Centrada en el Paciente}

Podemos entender que la atención al paciente oncológico ha pasado en una parte importante de las zonas culturales de un modelo Paternalista a uno de Atención 
Centrada en el Paciente con cáncer. Este cambio ha sido más destacable en occidente. Jayadevappa y Chhatre ${ }^{(20)}$ consideran que desde finales de los años 70, algunas organizaciones de salud, y otros estamentos, como las agencias de investigación, han apoyado la idea de la atención centrada en el paciente. La atención centrada en el paciente se ha convertido por lo tanto en una prioridad en el paciente con cáncer ${ }^{(21)}$. El Instituto de Medicina ${ }^{(3)}$ define dicha atención como el cuidado que respeta y responde a las preferencias individuales de los pacientes, sus necesidades y valores, y que asegura que las decisiones clínicas incorporan los valores de los pacientes. Este modelo de cuidados permite que haya flexibilidad en las relaciones entre los pacientes y los profesionales. En este sentido, Reeve et al., ${ }^{(22)}$ entienden que la atención centrada en el paciente apoya la participación activa del paciente en su cuidado.

El concepto de atención centrada en el paciente incluye la comunicación centrada en el Paciente $(\mathbf{C C P})^{(23)}$. Epstein et al., ${ }^{(12)}$ entienden que la CCP podría ser entendida como un rasgo (un estilo general de comunicación) o como un estado (conductas que se presentan durante una interacción particular), y también

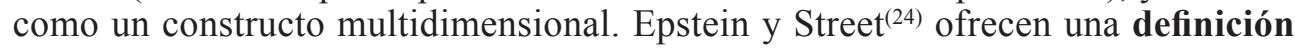
operacional de CCP en términos de un proceso de una interacción entre el paciente y el profesional:

1. Elicitando, comprendiendo y validando la perspectiva del paciente (ej. preocupaciones, sentimientos);

2. Comprendiendo al paciente dentro de su contexto psicológico y social;

3. Llegando a una comprensión compartida de los problemas del paciente y de su tratamiento (los pacientes y los profesionales tienen una idea común que es aceptada por el paciente y el profesional, sobre la enfermedad, las características del tratamiento y sus objetivos);

4. Ayudando al paciente a compartir el poder, a través de ofrecerle su involucración en elecciones relacionadas con su salud.

Estos autores consideran que la comunicación centrada en el Paciente CCP tiene seis funciones centrales:

1. Fomentar relaciones de curación;

2. Intercambiar información;

3. Responder a las emociones;

4. Manejar la incertidumbre;

5. Tomar decisiones;

6. Facilitar la autonomía del paciente

A partir de un metaanálisis, Venetis et al., ${ }^{(25)}$ concluyen que hay suficiente evidencia científica para apoyar la CCP.

\subsection{Ventajas de la CCP}

Carlson et al., ${ }^{(13)}$ y Reeve ${ }^{(22)}$ presentan resultados positivos en áreas clave de la atención al paciente oncológico, que han mostrado estar directa e indirectamente relacionados con una buena CCP: mayor satisfacción con los cuidados; mejor 
soporte por parte de los profesionales; mayor adherencia a los tratamientos, a los hábitos de salud y al autocuidado; decisiones clínicas de mayor calidad basadas en la evidencia; y que vayan en concordancia con los valores y las preferencias de los pacientes. También una sensación de mayor autoeficacia en el paciente, y un mejor ajuste psicológico. Y en conjunto, una mejor Calidad de Vida. En este sentido, el bienestar psicológico de los pacientes ha mostrado estar relacionado de forma positiva con aspectos de las consultas iniciales, como por ejemplo, que se les responda a sus preguntas durante las entrevistas ${ }^{(26)}$ o que el tono de la comunicación con el médico no sea de enfado ${ }^{(27)}$. Street et al., ${ }^{(28)}$ entienden que CCP contribuye a producir mejoras en la situación del paciente a través de diferentes vías, como son: la mejora en el acceso a los cuidados, aumentando los conocimientos del paciente, compartiendo la misma compresión de la situación, incrementando las alianzas terapéuticas y la autoeficacia del paciente.

\subsection{Comunicación y otros pro (Patient Reported Outcomes)}

En los párrafos siguientes vamos a presentar más en detalle la relación entre comunicación y algunas estas áreas PRO que han sido comentadas previamente. Vamos a indicar también si las áreas han sido incluidas en el cuestionario de comunicación de la EORTC.

\subsection{Comunicación y Calidad de Vida}

Una buena comunicación entre el paciente y el profesional ha demostrado tener una influencia importante en la Calidad de $\mathrm{Vida}^{(9)}$. Neumann ${ }^{(29)}$ indica que la empatía esta asociada con una mejor Calidad de Vida en tumores en diferentes localizaciones. Otros autores, como Farin et al., ${ }^{(30)}$, han concluido que los pacientes con cáncer que perciben a su médico como empático, tienen incrementos mayores en su Calidad de Vida tras la rehabilitación.

Aspectos de la comunicación se han evaluado en diferentes cuestionarios de Calidad de Vida de la EORTC. Los ítems incluidos en estos instrumentos evalúan principalmente la comunicación con otras personas que no son profesionales (ej familia, amigos, otras personas), comunicación en general, o contacto social en general. Se consideró que era necesario crear ítems que evaluaran las diferentes dimensiones de comunicación entre paciente y profesionales.

\subsubsection{Comunicación e información}

La comunicación y la información en Oncología son dos áreas que están muy relacionadas. Hack et al., ${ }^{(31)}$ diferencian entre la información que se ofrece a los pacientes y las habilidades de comunicación empleadas por los profesionales al dar dicha información. Entienden que un nivel bajo de habilidades de comunicación puede ser un obstáculo para una efectiva administración de información. En este sentido, estos autores consideran que una gran parte de las entrevistas entre el médico y el paciente se centran en discusiones relacionadas con la formulación de la enfermedad y el tratamiento, y que el éxito de estas discusiones depende en gran parte del grado en que las habilidades de comunicación ayuden al paciente 
a entender la enfermedad y el tratamiento. Estos autores concluyen que hace falta más investigación sobre las preferencias de los pacientes en el campo de las habilidades de comunicación relacionadas con la información.

Nuestro grupo decidió incluir en el cuestionario las habilidades que se emplean en la administración de información. El grupo de Calidad de Vida de la EORTC ha desarrollado el cuestionario de información EORTC QLQ-INFO25, que evalúa el contenido de la información que se ofrece (ej diagnóstico, tratamientos) o aspectos cualitativos, como el deseo de más información o la utilidad de esta. Estas áreas se decidieron no incluirlas en el cuestionario de comunicación ${ }^{(32)}$.

El cuestionario QLQ-INFO25 cuenta con la siguiente estructura:

- Contenidos de la información recibida: información sobre la enfermedad, pruebas médicas, tratamientos y otros servicios; información sobre otros aspectos: diferentes lugares donde recibir los cuidados, cosas que puedes hacer para ayudarte a ti mismo, información en otros formatos (escrita, información en $\mathrm{CD} /$ vídeo)

- aspectos cualitativos: satisfacción con la información recibida, deseo de recibir más información, de haber recibido menos información, utilidad global de la información recibida.

\subsection{Comunicación y satisfacción con los cuidados}

Las medidas de Satisfacción con los Cuidados suelen incluir algunas preguntas sobre satisfacción con la comunicación entre paciente y profesional. El grupo de Calidad de Vida de la EORTC cuenta con una escala de satisfacción, EORTC INPATSAT32 ${ }^{(33,34)}$. Nuestro grupo entendió que dichas medidas no cubren toda la evaluación sobre la comunicación entre paciente y profesional que se desea realizar. En este sentido, Feldman-Stewart et al., ${ }^{(36)}$ indican que las evaluaciones de satisfacción con los cuidados son medidas indirectas del éxito en la comunicación, y que ofrecen un insight limitado sobre qué debería ser cambiado para mejorar el proceso. Nuestro grupo decidió incluir el área de la satisfacción, como una sub-escala del cuestionario de comunicación.

\subsection{Diferencias culturales en comunicación}

Las diferencias culturales en aspectos clave del cuidado del paciente son un aspecto importante a tener en cuenta a la hora de ofrecer atención, y a la hora de crear instrumentos de medida PRO. En este sentido, se recogen diferencias culturales en satisfacción con los cuidados ${ }^{(36)}$, en información ${ }^{(37)}$, y en comunicación entre los pacientes y los profesionales ${ }^{(38)}$. Como ejemplo, en algunas culturas, tocar a los pacientes o mirarles a los ojos puede ser intrusivo. Además, en algunas culturas, los profesionales tienen un papel más activo que en otras cuando se comunican con los pacientes.

Estas diferencias en comunicación pueden estar relacionadas con el modelo de cuidados: en algunas culturas predomina el modelo Paternalista, en otras puede predominar un modelo basado en la Autonomía del Paciente. Además, el modelo de atención en algunas áreas, como en el Sur de Europa, puede estar cambiando en estos días. Estos cambios pueden influir en el estilo de comunicación entre pacientes y profesionales. 


\section{El Cuestionario de Comunicación de la EORTC}

\subsection{Objetivos del cuestionario}

El objetivo general del Grupo de Comunicación de la EORTC ha sido el de desarrollar y validar un cuestionario PRO (Patient Reported Outcomes) para pacientes con cáncer. Este cuestionario evalúa diferentes dimensiones de la comunicación entre los pacientes y los profesionales. La mayoría de su contenido se centra en las conductas de los profesionales. El Cuestionario de Comunicación de la EORTC se puede administrar solo o combinado con el cuestionario general de la Calidad de Vida de la EORTC (QLQ-C30).

Los aspectos culturales tienen un papel fundamental en el desarrollo del instrumento. El cuestionario intenta tener en cuenta las características diferenciales de las culturas que participan en su creación. En este sentido, se ha buscado desarrollar un cuestionario que pudiera ser empleado en diferentes culturas, y cuyos resultados en diferentes culturas se pudieran comparar.

El cuestionario se basa en el modelo de Comunicación Centrada en el Paciente CCP. En su Desarrollo se han tenido en cuenta cuatro de las seis funciones principales del modelo de la CCP propuestas por Epstein y Street ${ }^{(24)}$ : Fomentar relaciones de curación; Intercambiar información; Responder a las emociones; Manejar la incertidumbre. La función de toma de decisiones se ha considerado que requiere un cuestionario propio, y la del contenido de información, se entiende que puede estar cubierta con el cuestionario de información de la EORTC (QLQ-INFO25) ${ }^{(35)}$.

\subsection{Utilidad}

El objetivo del Grupo de Comunicación de la EORTC ha sido el de crear un cuestionario que pudiera ser empleado en diferentes campos: ensayos clínicos, investigación transcultural, en la práctica clínica diaria y en estudios clínicos.

En este sentido, el cuestionario puede ser empleado para evaluar nuevas intervenciones en el área de la comunicación como por ejemplo los programas de entrenamiento en comunicación ${ }^{(4)}$, las diferentes estrategias para comunicarse con pacientes con enfermedad avanzada, las nuevas vías de comunicación como el correo electrónico, y otros.

Puede ser útil para llevar a cabo investigación en diferencias culturales en comunicación. En este sentido, los métodos para mejorar la comunicación entre los pacientes y los profesionales deberían tener en cuenta las características de cada cultura.

El cuestionario también se puede emplear en la práctica clínica, para evaluar como el paciente considera la comunicación entre él y los profesionales que le tratan. Además, puede ayudar a ofrecer áreas de mejora en la comunicación de los profesionales. Los pacientes pueden tener varias entrevistas con los mismos profesionales, en las que se pueden realizar mejoras una vez que se ha realizado la evaluación inicial.

El cuestionario también se puede emplear en estudios clínicos en los que la comunicación entre los pacientes y los profesionales de un centro (o de un grupo de centros de un área) puede ser evaluada. También en estudios que valoren el impacto de la evaluación PRO sobre la comunicación entre el paciente y el profesional, en la práctica clínica diaria. 
Además, hay interés en realizar investigación en el área de la comunicación, sobre la relación con variables como la Satisfacción de los Cuidados, la Calidad de Vida y otras variables.

\subsection{Desarrollo del Cuestionario de Comunicación}

El desarrollo del Cuestionario de Comunicación sigue las Guías del Grupo de Calidad de Vida de la EORTC para la creación de cuestionarios ${ }^{(39)}$. El proceso de desarrollo se articula en cuatro fases:

(1) generación de un listado de áreas relacionadas con el área de comunicación:

(2) conversión de los aspectos de comunicación en preguntas;

(3) estudio internacional del modulo provisional;

(4) estudio internacional de validación a larga escala.

Todas las fases se realizan contando con la participación de centros provenientes de diferentes zonas culturales. Ello acrecienta la validez transcultural de los cuestionarios.

Las tres primeras fases del desarrollo del cuestionario de comunicación se han finalizado. El cuestionario se encuentra en la actualidad siendo estudiado en un estudio internacional (fase IV). A continuación queremos presentar un resumen de las tres primeras fases:

\section{Fase I}

En la fase I se generó un listado de aspectos relacionados con la comunicación entre los pacientes y los profesionales. Primero se realizó una revisión de literatura sistemática en bases de datos internacionales (PUBMED y PSYCHINFO). Además, se revisaron bases de datos de cuestionarios. Se evaluaron también otros estudios de expertos en el campo de la comunicación en Oncología que no se habían incluido en la presente revisión, al igual que otros trabajos identificados en los artículos de esta primera revisión. Se seleccionaron un total de 204 artículos y 79 instrumentos que se emplean para medir comunicación. Tras esta revisión de literatura se creó una lista inicial de 320 aspectos. Esta lista se redujo a 68 en base a los objetivos del cuestionario.

La visión que tienen los pacientes sobre la comunicación con los profesionales tiene un papel clave en el desarrollo del módulo. Por este motivo, se han realizado varias entrevistas con pacientes en esta y en otras fases del desarrollo del módulo. En esta fase I se han realizado entrevistas con pacientes y con profesionales en tres pasos:

1. Entrevistas semi-estructuradas con 23 pacientes provenientes de cuatro países: Austria, Croacia, Italia y España. En estas entrevistas se exploró su visión sobre el área de la comunicación, y los resultados se combinaron con la lista de áreas identificadas en la revisión de literatura.

2. Se han entrevistado a 23 profesionales de cinco países: Austria, Croacia, Noruega, España, y Taiwán

3. Se ha realizado una segunda ronda de entrevistas con 18 pacientes provenientes de cuatro países: Austria, Croacia, Italia, y España. 
En los pasos 2 y 3, se evaluó la lista provisional de áreas creada tras el primer grupo de entrevistas con los pacientes. Los pacientes que han participado en esta fase 1 se organizaban en seis grupos creados en base al estadio de la enfermedad (inicial y avanzado) y al estadío del tratamiento (inicial, durante y después). Había además, un séptimo grupo que estaban recibiendo cuidados paliativos. La lista provisional de aspectos relacionados con la comunicación se redujo en base a las entrevistas con los pacientes y los profesionales: se creó una lista provisional de 33 aspectos.

\section{Fase 2}

\section{Construcción del modulo provisional}

El listado provisional de aspectos relacionados con la comunicación, que han salido de la fase I, se convirtió en preguntas. Estas preguntas eran consistentes con el formato del QLQ-C30. No se incluyó la palabra “cáncer”, porque podría ser intrusiva para pacientes que no conocieran su diagnóstico ${ }^{(40)}$. Este módulo provisional fue revisado por profesionales del campo de la oncología, provenientes de Francia y España. Dichos profesionales encontraron que la expresión y el contenido de los ítems eran adecuadas.

\section{Fase 3}

En la fase III la lista provisional de ítems se ha evaluado en pacientes provenientes de 10 centros incluidos 5 áreas culturales (Norte y Sur de Europa, Inglaterra, Polonia y Taiwan). Estos pacientes se organizaban en siete grupos, en base a los criterios de: enfermedad inicial y avanzada; fase del tratamiento - antes, durante y después; más un séptimo grupo de pacientes que estaban recibiendo cuidados paliativos. Se han entrevistado a 140 pacientes en esta fase. Se realizaron entrevistas estructuradas con cada paciente sobre el contenido del cuestionario provisional. Se han realizado análisis cualitativos y cuantitativos. Hubo consistencia en las respuestas de los pacientes a través de las diferentes áreas culturales ${ }^{41}$.

\subsection{Cuestionario de Comunicación de la EORTC QLQ-COMU26: contenido y población a la que se dirige}

La versión revisada del cuestionario, QLQ-COMU26, tiene 26 ítems. Este cuestionario se estructura en 6 escalas y 4 ítems individuales (Tabla 1). Todas las escalas han presentado una fiabilidad adecuada en esta fase III (Alpha >0.78). El cuestionario se puede emplear con pacientes con cáncer en cualquier localización tumoral, en cualquier estadio de la enfermedad (inicial, avanzado - incluyendo a los cuidados paliativos) y de tratamiento (inicial, durante, seguimiento). No se ha evaluado en pacientes en fase final de la vida (pacientes que podemos entender como muy frágiles y con una esperanza de vida breve). Por lo tanto, de momento no se aplicaría a este subgrupo de pacientes.

El cuestionario permite a los pacientes indicar la categoría profesional que están evaluando: médico (s), enfermera (s), psicólogo (s), técnico (s) de radioterapia y otro (s) profesionales. Además, a los pacientes se les pide indicar el período específico de tratamiento que están evaluando (que no necesariamente tiene que ser el actual): diagnóstico, tratamiento o seguimiento. 
Tabla 1. Estructura del EORTC-COMU26.

\section{ESCALAS}

- conductas activas del paciente,

- aspectos de la relación entre el clínico y el paciente,

- las cualidades de un profesional creando una relación,

- las habilidades de los profesionales (lenguaje verbal - no verbal),

- el manejo del profesional de las emociones,

- las habilidades del profesional relacionadas con la administración de información.

\section{Items individuales}

- el profesional ha tenido en cuenta sus preferencias sobre cómo la información debería ser ofrecida,

- han corregido información que no han entendido bien,

- ha habido privacidad para hablar,

- satisfacción del paciente con la comunicación.

Fase 4 - Situación actual

El cuestionario EORTC QLQ-COMU26 está siendo estudiado en un estudio internacional amplio, fase IV del proceso de creación de escalas de la EORTC. Participan en él un número amplio de países de diferentes zonas culturales (Norte y Sur de Europa, India, Jordania, Taiwan y Australia). Este estudio va dirigido a confirmar las características psicométricas de sus escalas: su aplicabilidad y aceptación a través de diferentes culturas, la estructura de sus escalas, su fiabilidad, su validez y sensibilidad al cambio, y la invarianza de sus propiedades psicométricas a través de diferentes culturas. Una vez analizados los resultados de este estudio, podrían realizarse algunos cambios en el cuestionario.

\subsection{Obtencion del cuestionario actual de comunicación}

El cuestionario puede ser utilizado en su versión actual (aunque como decíamos, existe la posibilidad de que se realice más adelante algún cambio en su estructura). La versión en castellano se obtiene del Departamento de Calidad de Vida de la EORTC: se realiza un acuerdo de uso con ellos.

\section{Conclusiones}

El presente trabajo ha intentado hacer una presentación del área de la comunicación entre el paciente oncológico y los profesionales. Hemos visto la importancia de la comunicación en el soporte que se puede ofrecer a los pacientes. En la actualidad hay una necesidad de investigar en la comunicación entre pacientes y profesionales. Dicha investigación puede llevar a mejoras en la atención que se ofrece a los pacientes.

El cuestionario de comunicación de la EORTC QLQ-COMU26 es un instrumento PRO específico de cáncer para la evaluación de diferentes dimensiones de la comunicación entre el paciente y los profesionales. Ha sido desarrollado siguiendo 
un proceso detallado, en el que la visión de los pacientes y las características de diferentes culturas han sido tenidas en cuenta. Todo ellos entendemos que incrementa la validez de este instrumento.

\section{Agradecimientos}

El presente trabajo ha contado con la ayuda de becas del Grupo de Calidad de Vida de la EORTC (beca número EORTC QLG 006/2012), del Instituto Carlos III y FEDER, (proyecto número PI15/02114), y de la FUNDACIÓN CAJA NAVARRA.

\section{Referencias bibliográficas}

1. American Society of Clinical Oncology. Cancer care during the last phase of life. J Clin Oncol 1998;16:1986-96.

2. Committeee on Cancer Survivorship, Institute of Medicine. From cancer patient to cancer survivor: lost in transition. Washington: National Academics of Sciences, 2006.

3. Institute of Medicine. Crossing the quality chasm: a new health system for the $21 \mathrm{st}$ century. Washington, DC: National Academy Press, 2001.

4. Pham AK(1), Bauer MT, Balan S. Closing the patient-oncologist communication gap: a review of historic and current efforts. J Cancer Educ 2014;29:106-13. Doi: 10.1007/ s13187-013-0555-0.

5. Ansmann L, Kowalski C, Ernstmann N, Ommen O, Pfaff H. Patients ' perceived support from physicians and the role of hospital characteristics. Int J Qual Health Care 2012; 24: 501-8.

6. Epstein AS, Morrison RS. Palliative oncology: identity, progress, and the path ahead. Ann Oncol 2012; 23 Suppl 3: 43-8.

7. Fallowfield L. The challenge of interacting with patients in oncology. Eur J Cancer 2009; 45 Suppl 1: 445-6.

8. Moore PM, Rivera S, Grez M, Lawrie TA. Communication skills training for healthcare professionals working with people who have cancer. Cochrane Database Syst Rev 2013; Mar 28: 3.

9. Stewart M, Belle Brown J, Hammerton J, Donner A, Gavin A, Holliday RL, et al. Improving communication between doctors and breast cancer patients. Ann Fam Med 2007; 5: 387-94. Doi: 10.1370/afm.721

10. Fallowfield L, Jenkins V. Communicating sad, bad, and difficult news in medicine. Lancet 2004; 363: 312- 9. Doi:10.1016/S0140-6736(03)15392-5

11. Street RL Jr, Elwyn G, Epstein RM. Patient preferences and healthcare outcomes: an ecological perspective. Expert Rev Pharmacoecon Outcomes Res 2012; 12:167-80. Doi: 10.1586/erp.12.3.

12. Epstein RM, Franks P, Fiscella F, Shields CG, Meldrum SC, Kravitz RL, et al. Measuring patient-centered communication in patient-physician consultations: theoretical and practical issues. Soc Sci Med 2005; 61:1516-28. Doi:10.1016/j.socscimed.2005.02.001

13. Carlson LE, Feldman-Stewart D, Tishelman C, Brundage MD, SCRN Communication Team. Patient-professional communication research in cancer: an integrative review of research methods in the context of a conceptual framework. Psychooncology 2005; 14:812-28. Doi: 10.1002/pon.969 
14. Siviero P, Orrù G, Crepaldi G, Pegoraro R, Manzato E. Discussing end-of-life care issues with terminally ill patients and their relatives: comparisons among physicians, nurses and psychologists. Aging Clin Exp Res 2012; 24:35-42

15. Hack T. Psycho-oncology special issue on communication. Psychooncology 2005; 14: 797-8. Doi: 10.1002/pon.977

16. Carlson LE, Feldman-Stewart D, Tishelman C, Brundage MD; SCRN Communication Team. Patient-professional communication research in cancer: an integrative review of research methods in the context of a conceptual framework. Psychooncology 2005; 14 : 812-28. Doi: 10.1002/pon.969. Doi: 10.1002/pon.969

17. Croyle R. Foreword. En: Epstein RM, Street RL Jr, editors. Patient-Centered Communication in Cancer Care: Promoting Healing and Reducing Suffering. Bethesda, MD, USA: National Cancer Institute, 2007; p. 9.

18. Morgan M. The Doctor-Patient Relationship. En: Scambler G, editor. Sociology as applied to medicine. Edinburgh, New York: Saunders/Elsevier, 2008; p 49-65.

19. Parsons T. The social system. Glencoe, IL: Free Press, 1951.

20. Haidet P, Fecile ML, West H, Teal RC. Reconsidering the team concept: educational implications for patient-centered cancer care. Patient Educ Couns 2009; 77: 450-5. Doi: 10.1016/j.pec.2009.09.020.

21. Jayadevappa R, Chhatre S. Patient Centered Care - A conceptual model and review of the state of the art. Open Health Serv Policy J 2011; 4: 15-25.

22. Reeve BB, Thissen DM, Bann CM, Mack N, Treiman K, Sanoff HK, et al. Psychometric evaluation and design of patient-centered communication measures for cancer care settings. Patient Educ Couns 2017. Doi:10.1016/j.pec.2017.02.011. [Epub ahead of print]

23. Saha S, Mary CB, Cooper LA. Patient centeredness, cultural competence and healthcare quality. J Natl Med Assoc 2008; 100: 1275-85. Doi:10.1016/S0027-9684(15)31505-4

24. Epstein RM, Street RL Jr. Patient-centered communication in cancer care: promoting healing and reducing suffering. Bethesda, MD, USA: National Cancer Institute, 2007.

25. Venetis MK, Robinson JD, Turkiewicz KL, Allen M. An evidence base for patientcentered cancer care: a meta-analysis of studies of observed communication between cancer specialists and their patients. Patient Educ Couns 2009; 77:379-83. Doi: 10.1016/j. pec.2009.09.015

26. Butow PN, Devine R, Boyer M, Pendlebury S, Jackson M, Tattersall MH. Cancer consultation preparation package: changing patients but not physicians is not enough. $\mathrm{J}$ Clin Oncol 2004; 22: 4401-9. Doi: 10.1200/JCO.2004.66.155

27. Ong LM, Visser MR, Lammes FB, de Haes JC. Doctor-patient communication and cancer patients' quality of life and satisfaction. Patient Educ Couns 2000; 41:145-56. Doi: 10.1016/S0738-3991(99)00108-1

28. Street RL Jr, Makoul G, Arora NK, Epstein RM. How does communication heal? Pathways linking clinician-patient communication to health outcomes. Patient Educ Couns 2009;74:295-301.Doi: 10.1016/j.pec.2008.11.015

29. Neumann M, Wirtz M, Bollschweiler E, Mercer SW, Warm M, Wolf J, et al. Determinants and patient-reported long-term outcomes of physician empathy in oncology: a structural equation modelling approach. Patient Educ Couns 2007; 69: 63-75. Doi:10.1016/j. pec.2007.07.003

30. Farin E, Nagl M. The patient-physician relationship in patients with breast cancer: influence on changes in quality of life after rehabilitation. Qual Life Res 2012; 22:28394. Doi: $10.3109 / 09638288.2016 .1146360$ 
31. Hack TF, Degner LF, Parker PA; SCRN Communication Team. The communication goals and needs of cancer patients: a review. Psychooncology 2005; 14: 831-45. Doi: 10.1002/ pon.949

32. Arraras JI, Greimel E, Sezer O, Chie WC, Bergenmar M, Costantini A, et al. An international validation study of the EORTC QLQ-INFO25 questionnaire: an instrument to assess the information given to cancer patients. Eur J Cancer 2010; 46:2726-38. Doi: 10.1016/j.ejca.2010.06.118

33. Brédart A, Bottomley A, Blazeby JM, Conroy T, Coens C, D'Haese S, et al. An international prospective study of the EORTC cancer in-patient satisfaction with care measure (EORTC IN-PATSAT32). Eur J Cancer 2005;41:2120-31. Doi: 10.1016/j. ejca.2005.04.041

34. Arraras JI, Arias de la Vega F, Asin G, Arrazubi V, Manterola A, Morilla I. La evaluación de la satisfacción con los cuidados en el paciente oncológico. El sistema de medida de la EORTC. Psicooncología 2010; 7: 35-50.

35. Feldman-Stewart D, Brundage MD, Tishelman C; SCRN Communication Team. A conceptual framework for patient-professional communication: an application to the cancer context. Psychooncology 2005; 14: 801-9. Doi:10.1002/pon.950

36. Brédart A, Sultan S, Regnault A. Patient satisfaction instruments for cancer clinical research or practice. Expert Rev Pharmacoecon Outcomes Res 2010; 10: 129-41. Doi: 10.1586/erp.10.7.

37. Arraras JI, Greimel E, Chie WC, Sezer O, Bergenmar M, Costantini A, et al. Cross-cultural differences in information disclosure evaluated through the EORTC questionnaires. Psychooncology 2013;22:268-75. Doi: 10.1002/pon.2088

38. Surbone A. Cultural aspects of communication in cancer care. Support Care Cancer 2008;16:235-40. Doi: 10.1007/s00520-007-0366-0

39. Johnson C, Aaronson N, Blazeby J, Bottomley A, Fayers P, Koller M, et al. EORTC Quality of Life Group module development guidelines, fourth edn. Brussels: EORTC QL Group, 2011

40. Arraras JI, Kuljanic K, Sztankay M, Wintner LM, Costantini A, Chie WC et al. Initial phases in the development of a European Organisation for Research and Treatment of Cancer communication-specific module. Psychooncology 2015;24:236-40. Doi: 10.1002/ pon.3597

41. Arraras JI, Wintner LM, Sztankay M, Tomaszewski KA, Hofmeister D, Costantini A, et al. EORTC QLQ-COMU26: a questionnaire for the assessment of communication between patients and professionals. Phase III of the module development in ten countries. Support Care Cancer 2016 Dec 26. Doi: 10.1007/s00520-016-3536-0. [Epub ahead of print] 